\title{
Investigating and Treating Thyrotoxicosis
}

\section{Momin Shah, Simon Saunders \\ Warrington General Hospital}

$\underline{\text { AIM }}$

To evaluate the management of patients with hyperthyroidism against recommendations of the American Thyroid Association (ATA).

\section{METHODS}

The case records of all new patients referred with hyperthyroidism, 02.05.2009 - 01.04.2011, were analysed $(n=33)$, against recommendations of ATA.

\section{RESULTS}

$73 \%$ of the subjects were women. $24 \%$ patients had graves disease, $18 \%$ MNG, $18 \%$ thyroiditis, $15 \%$ "autoimmune hyperthyroidism", 15\% patients were broadly labelled as "hyperthyroid" (not otherwise specified), $3 \%$ patients had amiodarone induced thyrotoxicosis, $3 \%$ thyroid hormone resistance and $3 \%$ had subclinical hyperthyroidism. Carbimazole was used in all patients with graves on anti-thyroid medication (100\%). In patients treated with carbimazole, a baseline FBC was checked in $77 \%$ and LFTS in $51 \%$. Smoking history was documented in $64 \%$ of patients. Only $18 \%$ had documentary evidence of advice on smoking cessation. $0 \%$ of patients with grave or autoimmune thyrotoxicosis had TRAB levels re-checked prior to stopping carbimazole. 33\% of patients with MNG were referred for surgery (compressive symptoms) and the remainder $50 \%$ were referred for radio-iodine treatment. In patients with clinical presentation not diagnostic of graves disease only $16 \%$ had thyroid uptake scans. All patients with grave's disease who were referred for radio-iodine treatment were pre-treated with carbimazole (100\%).

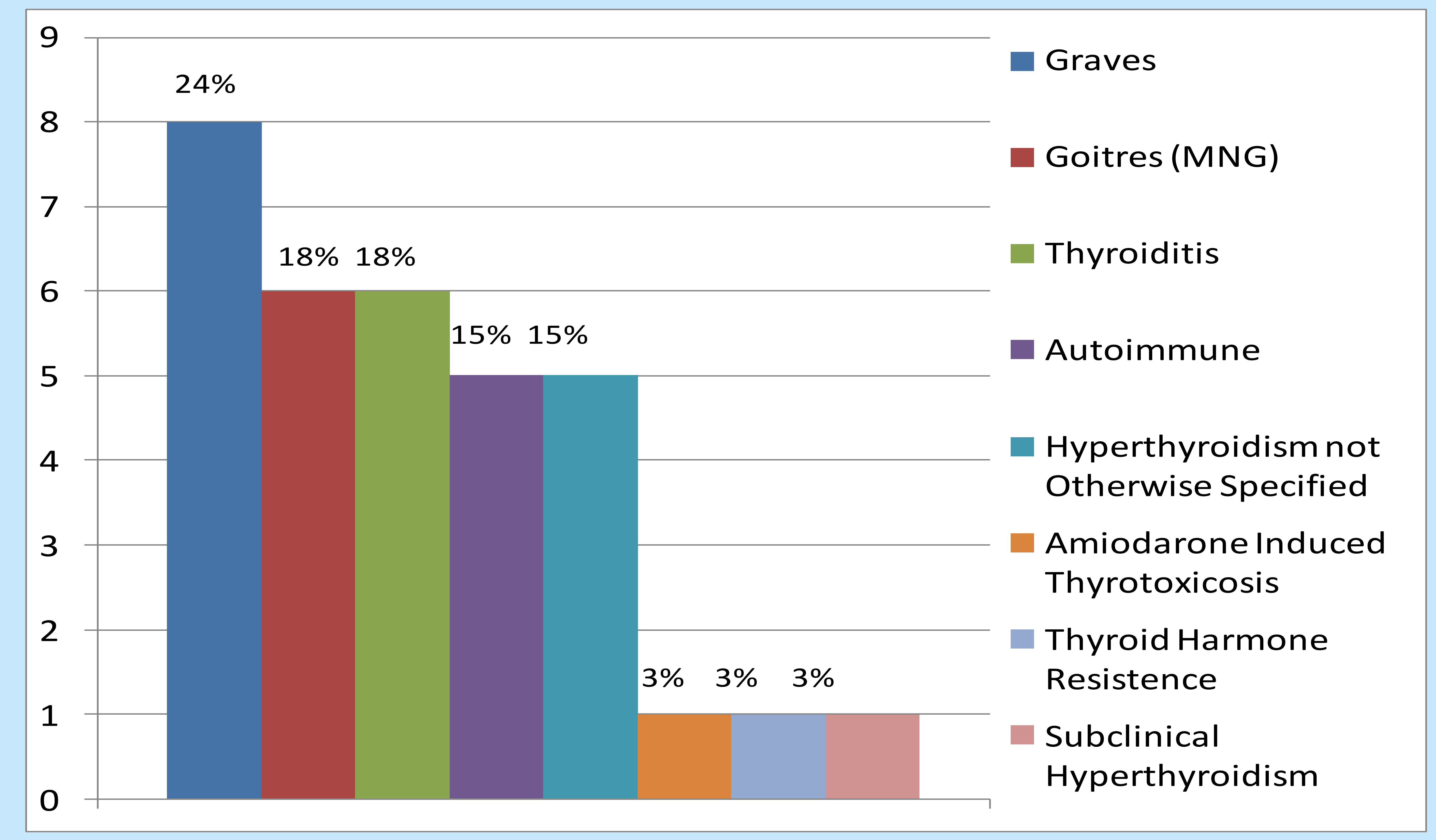

\section{DISCUSSION}

Anti-thyroid medication may rarely cause agranulocytosis and liver dysfunction therefore baseline FBC and LFTs should be checked in all patients. Graves opthamopathy is 6 times more common in smokers; risks of smoking should be discussed and documented. Patients with persistently raised TRAB levels should be counselled regarding the high chance of relapse and will benefit from more frequent monitoring of TFTs once off anti-thyroid medication. RAI uptake should be performed as part of evaluation when the clinical presentation of thyrotoxicosis is not diagnostic of graves disease. Finally "Hyperthyroidism" is not a diagnosis; every effort must be made to clarify the cause of hyperthyroidism. 